\title{
Targeted blockade of interleukin 9 inhibits tumor growth in murine model of pancreatic cancer
}

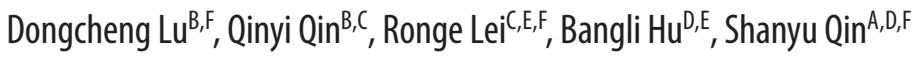 \\ First Affiliated Hospital of Guangxi Medical University, Nanning, China \\ A - research concept and design; $B$ - collection and/or assembly of data; $C$ - data analysis and interpretation; \\ $D$ - writing the article; $E$ - critical revision of the article; $F$ - final approval of the article
}

Address for correspondence

Shanyu Qin

E-mail: qsy0511@163.com

\section{Funding sources}

National Natural Science Foundation of China (grant No. 31360221)

Conflict of interest

None declared

Received on July 3, 2018

Reviewed on September 20, 2018

Accepted on February 18, 2019

Published online on October 23, 2019

Cite as

Lu D, Qin Q, Lei R, Hu B, Qin S. Targeted blockade of inter-

leukin 9 inhibits tumor growth in murine model of pancreatic cancer. Adv Clin Exp Med. 2019;28(10):1285-1292.

doi:10.17219/acem/104543

DOI

10.17219/acem/104543

Copyright

Copyright by Author(s)

This is an article distributed under the terms of the

Creative Commons Attribution Non-Commercial License

(http://creativecommons.org/licenses/by-nc-nd/4.0/)

\begin{abstract}
Background. Interleukin 9 (IL-9) has been implicated in the pathogenesis of several tumor types, but the role of anti-IL-9 in pancreatic cancer remains unclear.

Objectives. We aimed to explore the mechanism and effects of blockading IL-9 in a pancreatic cancer mouse model.

Material and methods. Panc02 cells were injected subcutaneously into mice to establish a mouse model. The mice were randomly categorized into 3 groups - the control group, the immunoglobulin $\mathrm{G}(\mathrm{lg} \mathrm{G})$ group and the anti-IL-9 group - corresponding to intravenous tail injection of phosphate-buffered saline (PBS), IgG isotype antibody and anti-IL-9 antibody, respectively. Then, the expression of IL-9, interleukin-9 receptor (IL-9r), Janus kinase 1 (Jak1), Jak3, and signal transducer and activator of transcription 3 (Stat3) mRNA was tested with quantitative reverse-transcription polymerase chain reaction ( $(R T$ T-PCR). Interleukin 9 in the tumor tissue was detected using enzyme-linked immunosorbent assay (ELISA). Western blotting and immunocytochemistry were performed to detect STAT3 and phosphorylation signal transducers and activators of transcription-3 (pSTAT3). Matrix metalloproteinase 2 (MMP2), MMP9 and vascular endothelial growth factor (VEGF) levels were assessed using immunocytochemistry.
\end{abstract}

Results. Tumor weight in the anti-IL-9 group was significantly lower than in the other groups $(p<0.05)$. There was a remarkable survival benefit in the anti-IL-9 group compared to the other groups $(p<0.05)$. The concentration of IL-9 in tumor tissue was significantly downregulated in the anti-IL-9-treated mice $(p<0.05)$. The expression of Jak1 and Jak3 mRNA and PSTAT3, MMP2 and MMP9 proteins in the anti-IL-9 group was lower than that of the PBS or lgG groups $(p<0.05)$, but the STAT3 and VEGF protein levels showed no significant difference $(p<0.05)$.

Conclusions. Anti-IL-9 antibody could effectively restrain the growth of pancreatic cancer in mice, and this effect may partly occur by blocking the STAT3 pathway.

Key words: pancreatic cancer, JAK2/STAT3 pathway, IL-9 antibody 


\section{Introduction}

Pancreatic cancer is one of the most aggressive metastatic malignancies, with a median survival rate of 6 months and a 5 -year survival rate of $5 \%$, and it ranks $4^{\text {th }}$ among cancer-related deaths in the USA., ${ }^{1,2}$ Surgery, chemotherapy and radiation therapy are the main treatment options for pancreatic cancer. However, the current treatment strategies are ineffective due to the fact that most of the patients are in advanced stages when they are first diagnosed. Therefore, finding novel therapeutic strategies is crucial to improve the effectiveness of treatment and to prolong the survival of pancreatic cancer patients.

Interleukin 9 (IL-9) is a multifunctional cytokine secreted by a host of pro-inflammatory immune cells, including Th2 cells, Th9 cells and Th17 cells. ${ }^{3,4}$ Interleukin 9 activates a heterodimeric receptor that comprises the IL-9 receptor $\alpha$-chain $(I L-9 r \alpha)$ and the $\gamma$-chain $(I L-9 r \gamma)$, and promotes the cross-phosphorylation of Janus kinase 1 (Jak1) and Janus kinase 3 (Jak3), which in turn induce the downstream activation of signal transducer and activator of transcription 3 (Stat3). ${ }^{5}$ Interleukin 9 has contrasting roles depending on the type of cancer. Studies have reported that while IL-9 impaired tumor cell growth in B16 melanoma and CT26 colon adenocarcinoma-tumorbearing mice, ${ }^{6}$ it could promote cancer growth and exhibit an anti-apoptotic function in various transformed cell lines. ${ }^{7,8}$ Thus, further research is needed in order to confirm the role of IL-9 in various cancers.

We have previously reported that IL-9 plays a tumorpromoting role in pancreatic cancer cell lines ${ }^{9,10}$ and therefore postulated that neutralizing IL-9 may exert a tumor-suppressing function in pancreatic cancer. In this study, we investigated the effect of the anti-IL-9 antibody on a subcutaneous Pan02 model and its potential mechanism. We report that anti-IL-9 antibody treatment can partly inhibit a pancreatic cancer tumor in vivo, which may provide an effective therapeutic strategy against pancreatic cancer. The study scheme is presented in Fig. 1.

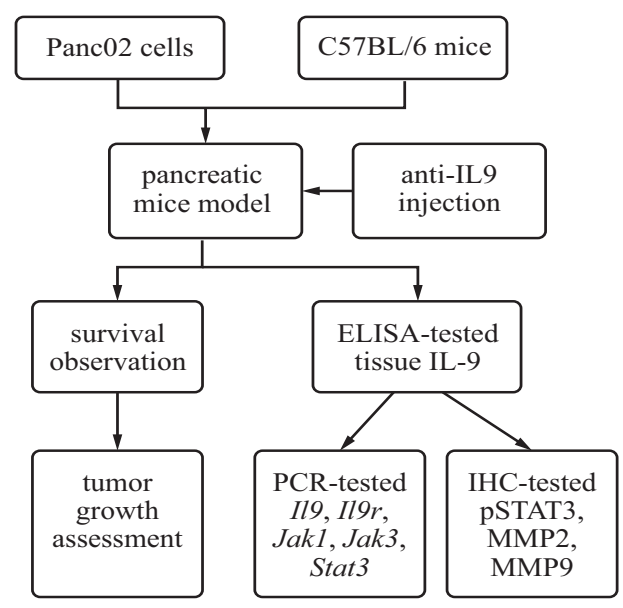

Fig. 1. The framework describing the idea of the study

\section{Material and methods}

\section{Cell culture}

Murine pancreatic adenocarcinoma cell line Panc02 was obtained from the American Type Culture Collection (ATCC) and cultured in Dulbecco's modified Eagle's medium (DMEM) (Gibco, Waltham, USA) supplemented with $100 \mathrm{mg} / \mathrm{mL}$ of streptomycin, $100 \mathrm{U} / \mathrm{mL}$ of penicillin and $10 \%$ fetal bovine serum (Gibco). The culture medium was replaced every $48 \mathrm{~h}$ unless otherwise described and the cells were incubated in $5 \% \mathrm{CO}_{2}$ and $95 \%$ air at $37^{\circ} \mathrm{C}$.

\section{Pancreatic cancer syngeneic model}

Forty-eight SPF-grade C57BL/6 mice (20-24 g), 5-6 weeks of age, were purchased from Hunan SJA Laboratory Animal Co. Ltd. (Changsha, China) and housed in the Animal Center of Guangxi Medical University, Nanning, China, under humidity- and temperature-controlled conditions with a 12-hour light/dark cycle. All procedures for handling the animals were approved by the Animal Experiments Ethics Committee of Guangxi Medical University. The cells were trypsinized and washed with phosphate-buffered saline (PBS) (consisting of potassium dihydrogen phosphate, disodium hydrogen phosphate, sodium chloride, and potassium chloride) (Solarbio, Beijing, China). Panc02 cells $\left(5 \times 10^{6}\right)$ in $200 \mu \mathrm{L}$ of PBS were subcutaneously injected into the right flank. Once the tumors reached about $50 \mathrm{~mm}^{3}$, the tumorbearing mice were tagged and divided into 3 groups (16 mice per group) and injected intravenously every 3 days for 18 days with sterile PBS, isotype control IgG (1 mg/kg) or anti-IL-9 antibody (1 mg/kg) (R\&D Systems, Minneapolis, USA).

\section{Tumor growth assessment}

The tumor diameter was measured with a caliper every other day and the volume was calculated using the following formula:

$(\text { short diameter })^{2} \times($ long diameter $) \times 0.5 .^{11}$

Eight mice from each group were sacrificed on the $5^{\text {th }}$ day after the last treatment. Individual tumors from the mice were weighed and dissected for subsequent study. The inhibition rate was calculated using the following formula, in which A corresponds to the average tumor weight in the control group and B corresponds to tumor weight in the treated groups:

$$
(\%)=[(\mathrm{A}-\mathrm{B}) / \mathrm{A}] \times 100 \% .
$$

\section{Survival observation}

Surrogate endpoints for survival were used: either when the tumors reached an average diameter of $20 \mathrm{~mm}$ or when they became ulcerated. ${ }^{12,13}$ The survival time of the remaining mice ( 8 mice per group) was recorded at the end 
of the observation period. The prolonged rate of survival time was calculated using the following formula, in which A corresponds to the average tumor weight in the control group and B corresponds to the average tumor weight in the treated groups:

$$
(\%)=[(\mathrm{B}-\mathrm{A}) / \mathrm{A}] \times 100 \% \text {. }
$$

During treatment with anti-IL-9 antibody, we also observed whether the mice displayed abnormal behavior, such as an altered mental state or weight loss.

\section{RNA isolation and qRT-PCR}

The total RNA was extracted from tumor tissues using an RNAiso plus Kit (TaKaRa, Beijing, China). cDNA synthesis was performed using a PrimeScript ${ }^{\mathrm{TM}} \mathrm{RT}$ reagent Kit (TaKaRa). Real-time polymerase chain reaction (RT-PCR) was performed with PrimeScript ${ }^{\mathrm{TM}}$ RT PCR Master Mix according to the manufacturer's protocol. The forward and reverse primers used for PCR were as follows:

IL-9 forward 5'ATG TTG GTG ACA TAC ATC CTT GC-3' and reverse 5'-TGA CGG TGG ATC ATC CTT CAG-3';

IL-9r forward 5'-GTA TTT ACA GGA TCG ACT GCC AC-3' and reverse 5'-CCC AGA AGG TGC ATT TGT GTT-3'; Jak1 forward 5'-GGA GTA CTA CAC AGT CAA GGA CGA-3' and reverse 5'-AAA CAT TCC GGA GCG TAC C-3'; Jak3 forward 5'-CCA GAC CAG CAG AGG GAC TT-3' and reverse 5'-CCA AAG CGA ACA GCA GTA GGC-3'; Stat3 forward 5'-GTT CCT GGC ACC TTG GAT T-3' and reverse 5'-CAA CGT GGC ATG TGA CTC TT-3'.

The relative expression was normalized to GAPDH and gene expression was analyzed using the $2-\Delta \Delta \mathrm{CT}$ method.

\section{Immunohistochemical analysis}

The tumor tissues were formalin-fixed and paraffinembedded, and specimen sections $4 \mu \mathrm{m}$ in thickness were prepared. The slides were treated in an autoclave oven for 5-10 min to unmask the heat-induced antigen and they were blocked with 5\% goat serum in PBS for $1 \mathrm{~h}$. The sections were incubated with primary antibodies against STAT3 (Cat\# ab68153, 1:500, Abcam, Cambridge, UK), pSTAT3 (Cat\# ab76315, 1:1000, Abcam), matrix metalloproteinases (MMPs) 2 and 9 (Cat\# BM4098 and BM4075, 1:500, Boster Biological Technology, Pleasanton, USA), and vascular endothelial growth factor A (VEGF A) (Cat\# M00045, 1:400, Boster Biological Technology) at $4^{\circ} \mathrm{C}$ overnight, followed by the secondary antibody (Cat\# A-21074, Zymed, Waltham, USA) at room temperature for $30 \mathrm{~min}$. Diaminobenzidine (DAB) was used to facilitate the locating of the antibody. Finally, the slides were rinsed in PBS and then counterstained with hematoxylin. The stained sections were captured using an Olympus DP72 microscope (Olympus Corporation, Shinjuku, Tokyo, Japan). The positively-labeled cells and negative cells in 5 randomly selected fields were counted at $\times 200$ magnification by 2 independent observers and the mean percentage of positive cells was calculated using Image-Pro Plus v. 6.0 (Media Cybernetics, Rockville, USA).

\section{Western blot analysis}

The proteins were collected from tumor tissues using RIPA lysis buffer (consisting of sodium chloride, tris(hydroxymethyl)aminomethane, sodium deoxycholate, and NP-40 lysis buffer) containing protease inhibitors and phosphatase inhibitors (Roche, Basel, Switzerland). The lysates were subjected to centrifugation at $12,000 \mathrm{rpm}$ for $20 \mathrm{~min}$ at $4^{\circ} \mathrm{C}$. The total protein was then measured with Pierce BCA assay (Solarbio). One part of $5 \times$ sample buffer and 4 parts of equal concentrations of protein were mixed and heated at $95^{\circ} \mathrm{C}$ for $10 \mathrm{~min}$. Equal amounts of lysates were separated with $10 \%$ sodium dodecyl sulfate-polyacrylamide gel electrophoresis for $90 \mathrm{~min}$ and were blotted onto a polyvinylidene fluoride (PVDF) membrane (Merck Millipore, Burlington, USA). The membranes were blocked with $1 \times$ TBS containing $0.1 \%$ Tween 20 and $2 \%$ BSA at room temperature for $1 \mathrm{~h}$ and subsequently incubated with rabbit monoclonal anti-STAT3 (phospho-Y705; 1:2,000; Abcam) at $4^{\circ} \mathrm{C}$ overnight, followed by secondary fluorescent antibodies labeled with LI-COR IRDye 680 (LI-COR Biosciences, Lincoln, USA) at room temperature for $1 \mathrm{~h}$. The signals were determined using Odyssey Infrared Imaging System and quantitated with LICOR imaging software (LI-COR Biosciences).

\section{ELISA for IL-9}

The tumor tissues were washed and homogenized in cold PBS before being stored at $-20^{\circ} \mathrm{C}$ overnight. The tumor homogenates had undergone 2 freeze-thaw cycles and were subsequently centrifuged at $5,000 \mathrm{~g}$ for $5 \mathrm{~min}$ at $4^{\circ} \mathrm{C}$. Interleukin 9 levels in the supernatant were determined using enzyme-linked immunosorbent assay (ELISA) Kits according to the manufacturer's instructions (Cusabio, Wuhan, China). The optical density was measured at $450 \mathrm{~nm}$ with a standard curve range of $15.6 \mathrm{pg} / \mathrm{mL}$ to $1,000 \mathrm{pg} / \mathrm{mL}$.

\section{Statistical analysis}

The data is expressed as mean values \pm standard deviation (SD). All data was analyzed with SPSS v. 17.0 software (SPSS Inc., Chicago, USA). Comparisons between 2 groups were carried out using Student's t-test while comparisons among multiple groups were analyzed using the one-way analysis of variance (ANOVA) method followed by the least significant differences (LSD) post hoc test. The $X^{2}$ test and Fisher's exact probability test were used to compare enumeration data. Survival analysis was performed using the Kaplan-Meier method and the log-rank test. Differences in means were considered statistically significant when $\mathrm{p}<0.05$. 


\section{Results}

\section{Blockading IL-9 slows in vivo pancreatic tumor growth}

The tumors in the anti-IL-9 group showed significantly slower growth rates than those in the PBS and IgG groups ( $p<0.05$; Fig. 2A). Significant differences in tumor volume among the 3 groups were observed on day 17, whereas no significant differences between the PBS group and the IgG group were found. Additionally, tumor weight was significantly lower in the anti-IL-9 group than in both the PBS and IgG groups ( $\mathrm{p}<0.05$; Fig. 2B). At the end of the experiment, the anti-IL-9 group achieved $24.2 \%$ tumor growth inhibition ( $\mathrm{p}<0.05$ vs the control group). These results suggest that the anti-IL-9 antibody can inhibit tumor growth.

\section{Neutralizing IL-9 extended survival time in a murine model}

Measuring the time from Panc02 cell injection until the surrogate endpoint for survival, we found that administering anti-IL-9 significantly prolonged the survival of tumor-bearing mice compared to the PBS- or IgG-administered groups ( $<<0.05$; Fig. 3 ): the increase in survival time among the anti-IL-9 group was $16.8 \%$. Additionally, exposure to anti-IL-9 treatment did not lead to abnormal behavior or dramatic changes in the body weight of these animals, implying its tolerability during a sustained period of administration.

\section{Administering anti-IL-9 antibody decreased IL-9 concentration in tumor tissue}

To determine whether the anti-IL-9 antibody treatment blocked IL-9 activity, the concentration of IL-9 in the tumor tissue was detected with ELISA. We observed a significant reduction in IL-9 activity in the antiIL-9-treated mice compared to the PBS-treated and IgGtreated mice ( $<<0.05$; Fig. 4A). Similarly, quantitative reverse-transcription polymerase chain reaction (qRTPCR) analysis suggests that the expression of $I L-9$ mRNA was significantly lower after anti-IL-9 treatment $(\mathrm{p}<0.05$; Fig. 4B).

\section{Treating with anti-IL-9 antibody alters the JAK-STAT pathway in tumor-bearing mice}

Because IL-9-mediated signal transduction activated molecular members of the STAT $3,^{5}$ MAPK and PI3K pathways $^{14}$ - which were potentially involved in the activity of the IL-9-IL-9R axis - we postulated that the blockade of IL-9 may mediate the JAK/STAT pathway in pancreatic-tumor-bearing mice. As is shown in Fig. 5, the relative expression of Jak1 and Jak3 mRNA

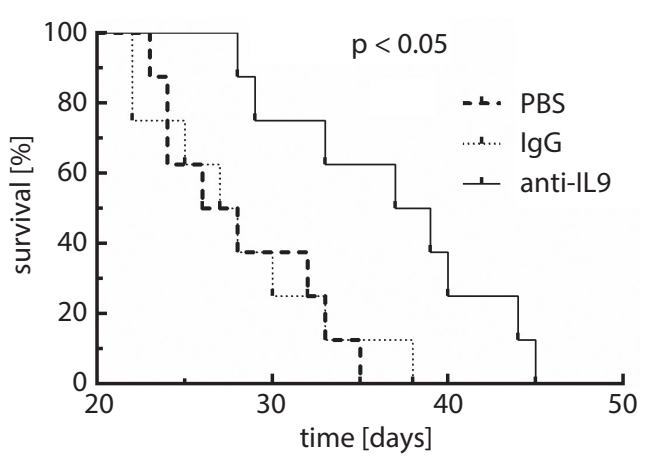

Fig. 3. Treatment with anti-IL-9 antibody extends the survival of mice Tumor growth exceeding $20 \mathrm{~mm}$ or at signs of ulcerated was used as a surrogate for survival. Kaplan-Meier analysis demonstrates that the survival time ( $n=8 /$ each) was significantly prolonged in anti-IL-9treated mice compared to PBS- or lgG-treated mice ( $p<0.05)$. Moreover, the mice experienced no apparent toxicity during the treatment
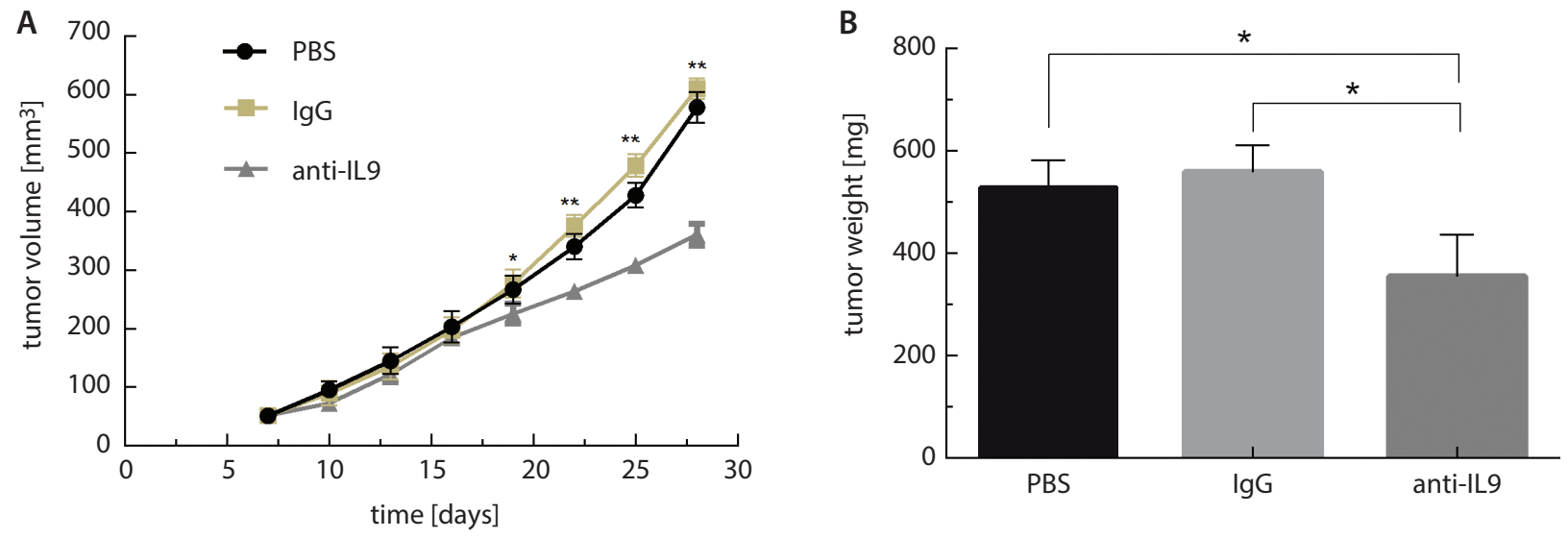

Fig. 2. Anti-IL-9 treatment results in slowed tumor growth in vivo. When tumors reached approx. $50 \mathrm{~mm}^{3}$, mice were randomly separated into 3 cohorts: PBS group, IgG group and anti-IL-9 group, and tumor growth was monitored. A - mice treated with anti-IL-9 antibody showed significant delay in tumor growth as compared to control groups $(\mathrm{p}<0.05)$. Each data point represents average tumor volume $(\mathrm{n}=8 / \mathrm{each})$ and error bars denote standard error; $\mathrm{B}$ - comparison of weight of tumor in different groups ( $n=8 /$ each) 
in the anti-IL-9 group was markedly lower than that of the PBS or IgG groups ( $<<0.05$; Fig. 5B,C), whereas the mRNA levels of $I L-9 r$ and Stat3 did not show any difference across all 3 groups ( $p>0.05$; Fig. 5A,D). Similarly, the western blot results showed that IL-9 inhibition failed to suppress STAT3 expression in the anti-IL-9 group ( $\mathrm{p}>0.05$; Fig. 6A,B), but pSTAT3 expression was effectively decreased in the tumor tissues from the antiIL-9 group compared to those treated with PBS or IgG antibody ( $<<0.05$; Fig. 6A,C). Consistent with the above results, the immunohistochemistry results revealed that tumor tissues in the anti-IL-9 group expressed significantly less PSTAT3 protein when compared to the other groups ( $<<0.05$; Fig. 7).
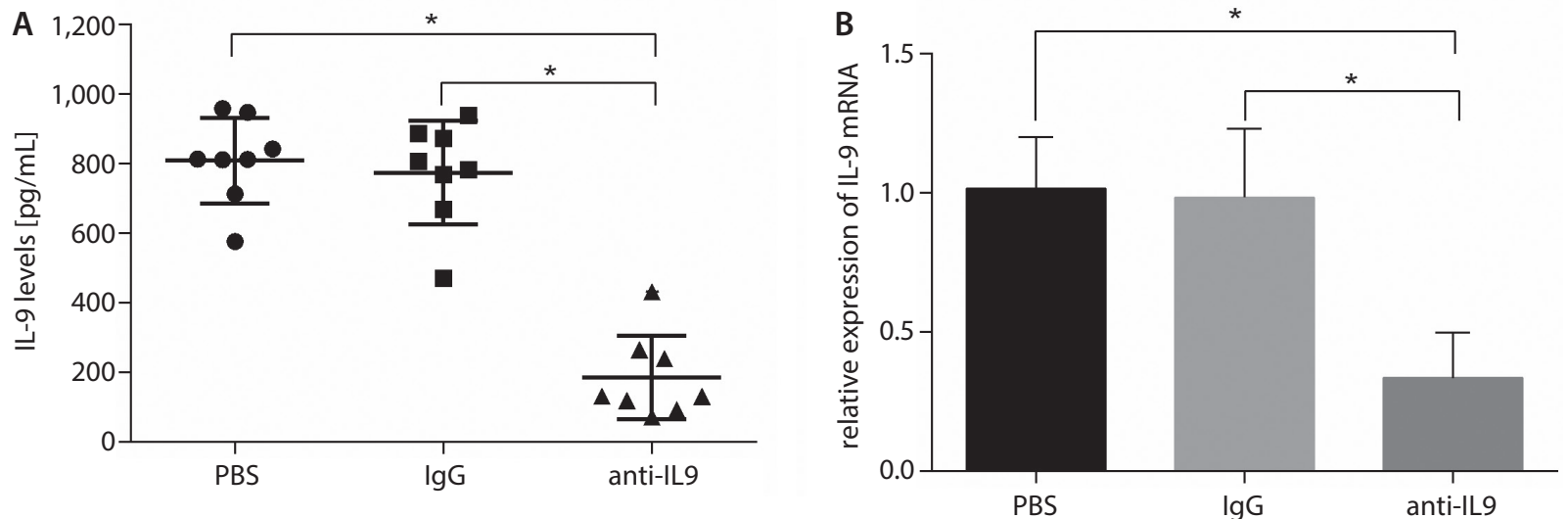

Fig. 4. Comparison of level of IL-9 in anti-IL-9 antibody, PBS and IgG-treated groups $(n=8 /$ each). The data confirmed antibody neutralization of IL-9 had a significant effect on the production of IL-9. A - concentration of IL-9 in tumor tissue was detected using ELISA; B - the expression of IL-9 mRNA was detected using qRT-PCR

${ }^{*} p<0.05$ compared with PBS or lgG control group; $n s-p>0.05$ compared with PBS or lgG control group. Data is shown as mean \pm SD.

A

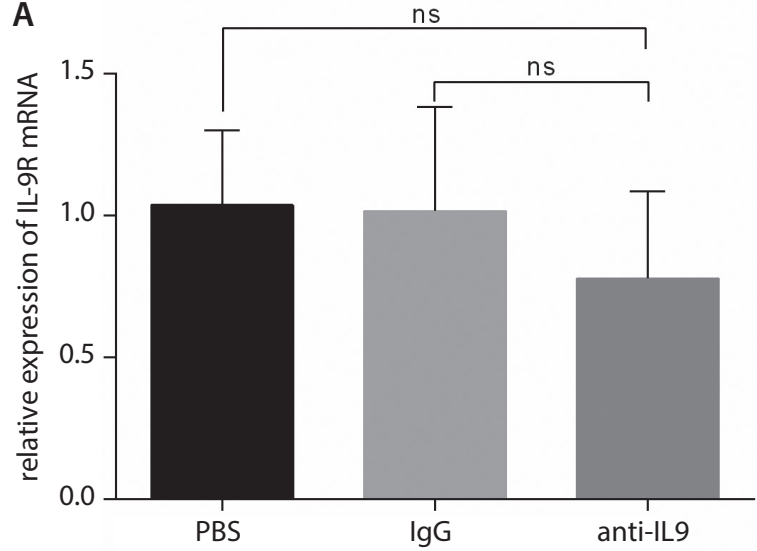

C

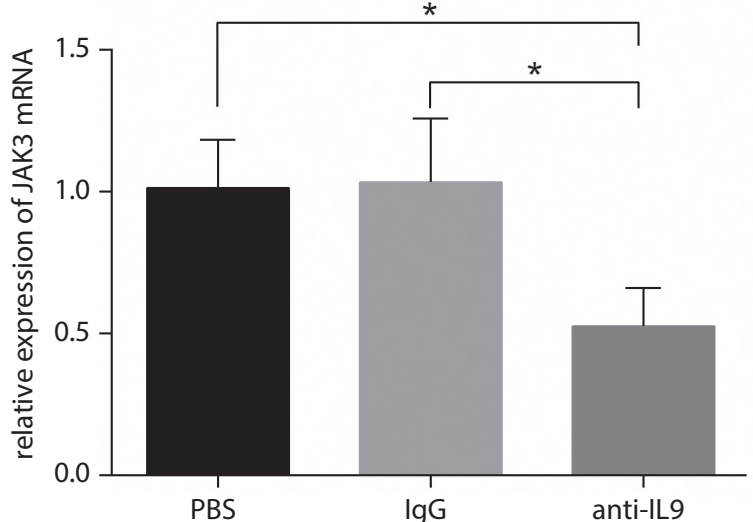

B

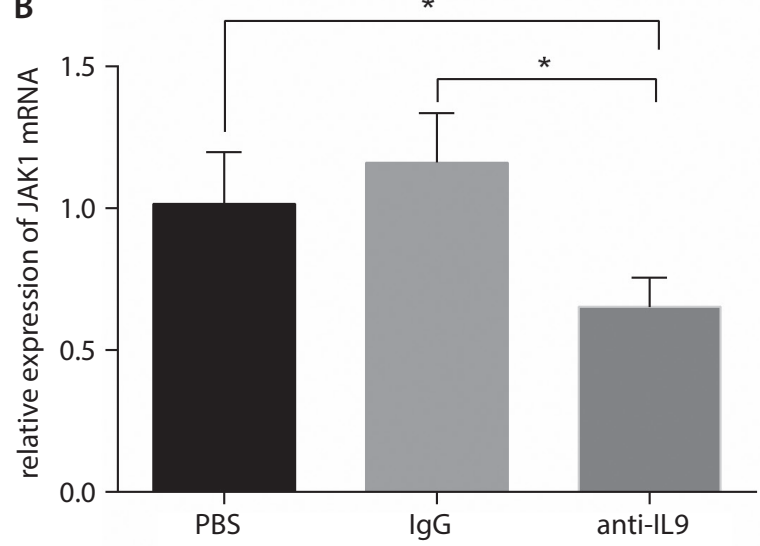

D

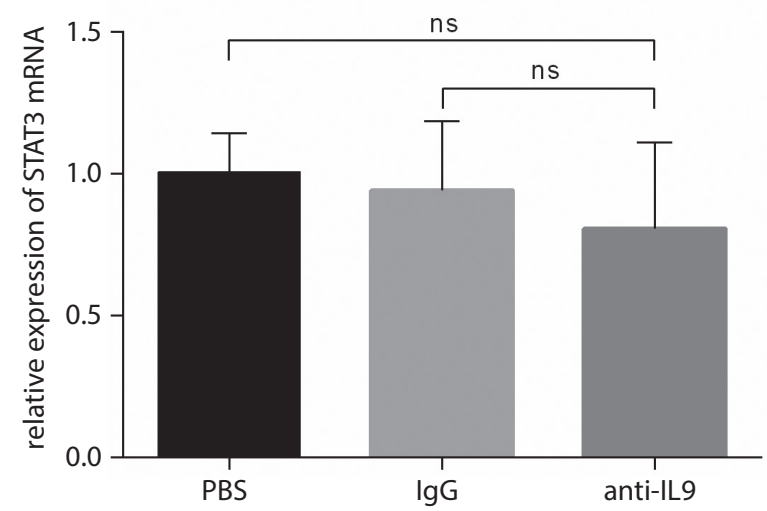

Fig. 5. The mRNA level of IL-9r, Jak1, Jak3, and Stat3 in mice with administration of PBS, IgG and anti-IL-9. A-D - relative expression of IL-9r, Jak1, Jak3, and Stat 3 was detected using qRT-PCR.

${ }^{*} \mathrm{p}<0.05$ compared with control PBS- or lgG-treated mice; $n s-p>0.05$ compared with PBS or lgG control group. Data is presented as mean \pm SD. 
A

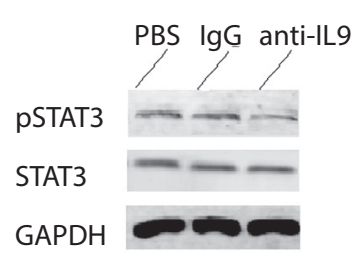

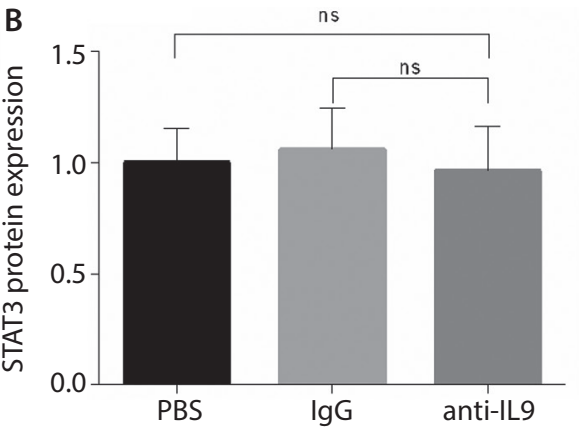

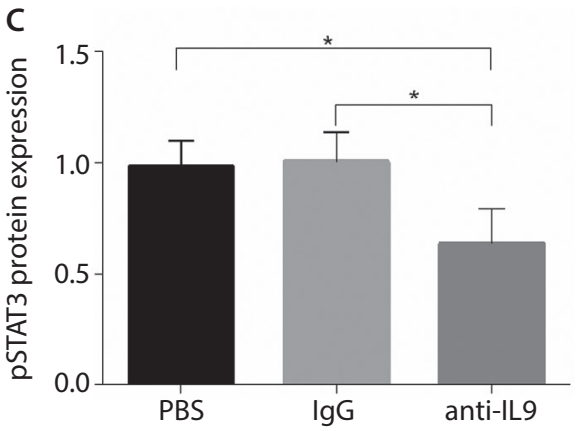

Fig. 6. Administration of anti-lL-9 antibody suppressed STAT3 phosphorylation in tumor tissue. A - expression of STAT3 and pSTAT3 was detected using western blot. B,C - western blot analysis indicated that the protein levels of PSTAT3 in anti-IL-9 group were significantly downregulated, but no significant changes in level of STAT3 protein was found

${ }^{*} p<0.05$ compared with PBS or lgG control group. Data is shown as mean \pm SD.

A

anti-IL9

$\lg G$

PBS

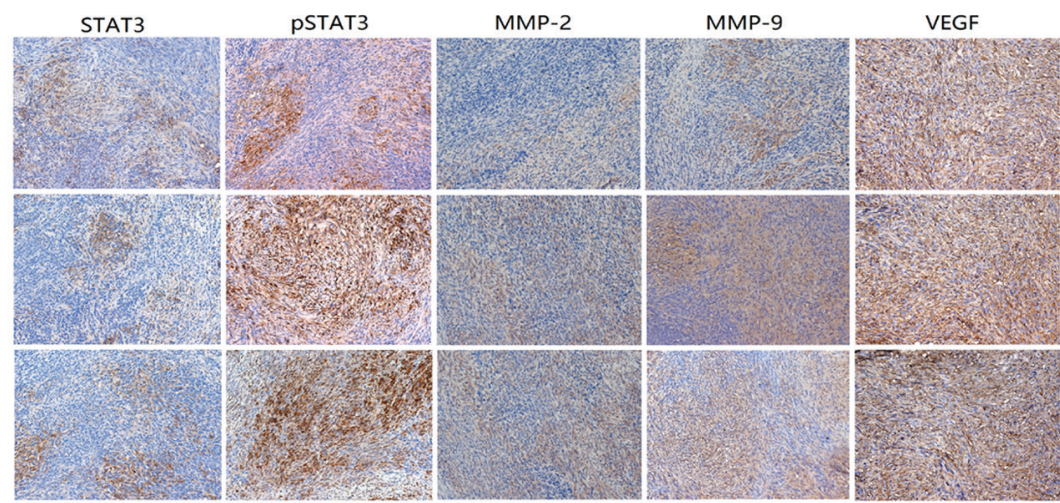

B

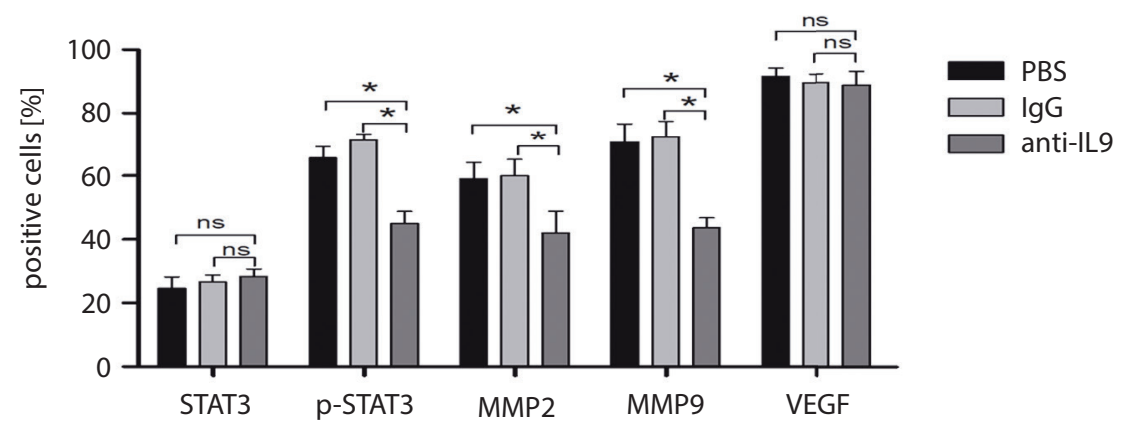

Fig. 7. The effect of anti-IL-9 antibody treatment on the expression of STAT3, pSTAT3, MMP2, MMP9, and VEGF in subcutaneous tumor. A - immunohistochemistry detected significantly less PSTAT3 (brown, nuclei), MMP2 (brown, cytoplasm) and MMP9 (brown, cytoplasm) in the tumors of anti-IL-9-treated mice than in those of PBS or lgG-treated mice. No significant changes was found in the levels of STAT3 (brown, cytoplasm) and VEGF (brown, cytoplasm) between groups; B - the semi-quantitative analysis of the above results

${ }^{*} p<0.05$ compared with PBS or IgG control group. All images are presented at $\times 200$ magnification.

\section{Treating with anti-IL-9 antibody decreases STAT3-regulated downstream target products}

Next, we determined whether suppressing pSTAT3 with the anti-IL-9 antibody could initiate the suppression of STAT3-regulated genes, MMP 2 and 9 and VEGF. ${ }^{15}$ As shown in Fig. 7, the tumor cells and stroma showed strong reactivity to the antibodies for STAT3, pSTAT3, MMP 2 and 9, and VEGF. Our immunohistochemistry results showed a significant reduction in pSTAT3-, MMP2- and MMP9-positive cells ( $<$ < 0.05; Fig. 7), whereas the number of STAT3- and VEGF-positive cells was not significantly different in the anti-IL-9 group compared to the PBS and IgG groups ( $p>0.05$; Fig. 7 ). We concluded that anti-IL-9 antibody may inhibit pancreatic cancer growth by downregulating JAK/STAT3 signaling.

\section{Discussion}

Immune cells and the cytokines secreted by them are involved in a multitude of physiological and pathological processes. ${ }^{16,17}$ Interleukin 9, a pleiotropic cytokine secreted by a number of immune cells, is implicated in the pathogenesis of several diseases and conditions. ${ }^{5,6}$ Interleukin 9 facilitates an immunosuppressive environment to promote tumor growth or to restrain tumor progression based on tumor type. ${ }^{18}$ Interleukin 9 was shown to inhibit the growth of HTB-72 melanoma cells and it favors 
the survival of $\mathrm{CD} 4{ }^{+} \mathrm{CD} 8^{+}$double-positive T cells; it also displays cytotoxic activity against melanoma cells. ${ }^{19} \mathrm{How}-$ ever, IL-9 also substantially contributes to the proliferation and migration of lung cancer cells while helping them escape apoptosis. ${ }^{20}$ Similar biological functions of IL-9 were also observed in breast cancer, ${ }^{18}$ colon carcinoma ${ }^{18}$ and hematopoietic malignancies, such as diffuse large B-cell lymphoma (DLBCL). ${ }^{8}$ Moreover, IL-9 can protect DLBCL cell lines LY1 and LY8 from prednisolone and rituximab, whereas the effect of IL-9 on DLBCL cell lines could be impaired and reversed through the knock-down of IL-9R. ${ }^{8}$ Because IL-9 is strongly associated with tumor progression, blocking IL-9 may be an applicable strategy for tumor treatment. Smith et al. showed that the neutralization of IL-9 in conjunction with CpG-ODN injection could induce tumor rejection in BALB-neuT- and MUC1-tolerant tumor models. ${ }^{21}$ Anti-IL-9 treatment inhibited tumor growth in WT mice with breast cancer cell line $4 \mathrm{~T} 1{ }^{18}$ In this setting, we investigated whether targeting IL-9 could influence pancreatic cancer progression.

A murine Panc02 model is widely used in pre-clinical study, and immunocompetent C57BL/6 mice provide a tumor microenvironment similar to a real immune environment. ${ }^{22,23}$ A significantly slower tumor growth rate and lower tumor weight were observed in the anti-IL-9 group compared to the PBS or IgG groups, indicating that the antiIL-9 antibody could limit the growth of pancreatic cancer.

Next, we investigated the effect of anti-IL-9 treatment on survival. The results showed that neutralizing IL-9 significantly prolonged the survival time in Panc02-tumorbearing mice. Notably, we observed that tumor growth occurred constantly in the survival period after the last administration of anti-IL-9, suggesting that the anti-IL-9 antibody could inhibit tumor growth only in a period of constitutive anti-IL-9 antibody injection, but failed to provide a long-term solution to inhibit tumor growth completely. In addition, the concentration of IL-9 in tumor tissue from the anti-IL-9 group was markedly reduced. Taken together, our results suggest that neutralizing IL-9 could inhibit the growth of pancreatic cancer in vivo by inhibiting IL-9.

The STAT3 signaling is involved in the regulation of the cell cycle, apoptosis, angiogenesis, metastasis, and immune evasion across a variety of tumor types, including pancreatic cancer. ${ }^{24-26}$ Notably, sustained activation of STAT3 at phosphorylated Tyr705 exists in 30-100\% of human tumor specimens and in several pancreatic cancer cell lines. ${ }^{27}$ Moreover, STAT3 was previously reported to be potentially involved in the IL-9-IL-9R axis, and IL9 -induced receptor activation promotes phosphorylation of JAK1 and JAK3, which leads to the downstream activation of the STAT3 pathway. ${ }^{5,14,28}$ Dysregulation of IL-9 in vitro could lead to autonomous cell growth and malignant transformation of lymphoid cells, which was strongly related to constitutive activation of the JAK/STAT pathway. ${ }^{29}$ Thus, we postulate that an IL-9 blockade may affect the STAT3 pathway. Consistent with this hypothesis, our results showed a dramatic reduction of JAK1, JAK3 and pSTAT3 in the tumor tissue from the mice injected with the anti-IL-9 antibody, so we concluded that the STAT3 pathway may be implicated in this process.

The activation of STAT3 has been reported to induce MMP9 expression ${ }^{30}$ and to upregulate VEGF by directly binding to the VEGF promoter, ${ }^{31}$ thereby facilitating cell invasion. In this study, we further examined whether the alteration of STAT3 could influence the expression of MMP9 and VEGF. We observed that the expression of MMP2 and MMP9 was reduced after the administration of the anti-IL-9 antibody, but no significant change in the expression of VEGF was observed among the 3 groups, implying that the inhibition of the STAT3 pathway caused by anti-IL-9 could further suppress the expression of MMP2 and MMP9, but had no effect on VEGF expression.

Although our study elucidated the effect of anti-IL-9 in pancreatic cancer and its possible underlying mechanism, there were several limitations to this study. The pancreatic cancer mouse model was established using Panc02 cells; the effect and mechanism of IL-9 on pancreatic cancer should be verified in a mouse model established by the use of other pancreatic cancer cells. Although we showed an anti-IL-9-mediated STAT3 pathway inhibition in pancreatic cancer, a rescue experiment using STAT3 pathway inhibitors is needed to verify whether the pathway is truly involved in this process.

\section{Conclusions}

Our study demonstrated that treating pancreatic-tumorbearing mice with the anti-IL-9 antibody can delay tumor growth, possibly via the inhibition of the STAT3 signaling pathway. Further experimentation using other pancreatic cancer mouse models is required to validate these results.

\section{References}

1. Siegel RL, Miller KD, Jemal A. Cancer statistics, 2017. CA Cancer J Clin. 2017;67(1):7-30.

2. Hidalgo M. Pancreatic cancer. N Engl J Med. 2010;362(17):1605-1617.

3. Stassen M, Schmitt E, Bopp T. From interleukin-9 to T helper 9 cells. Ann N Y Acad Sci. 2012;1247:56-68.

4. Schmitt E, Klein M, Bopp T. Th9 cells, new players in adaptive immunity. Trends Immunol. 2014;35(2):61-68.

5. Noelle RJ, Nowak EC. Cellular sources and immune functions of interleukin-9. Nat Rev Immunol. 2010;10(10):683-687.

6. Rivera Vargas T, Humblin E, Vegran F, Ghiringhelli F, Apetoh L. TH9 cells in anti-tumor immunity. Semin Immunopathol. 2017;39(1):39-46.

7. Chen J, Petrus M, Bryant BR, et al. Autocrine/paracrine cytokine stimulation of leukemic cell proliferation in smoldering and chronic adult T-cell leukemia. Blood. 2010;116(26):5948-5956.

8. Lv X, Feng L, Ge X, Lu K, Wang X. Interleukin-9 promotes cell survival and drug resistance in diffuse large B-cell lymphoma. J Exp Clin Cancer Res. 2016;35(1):106.

9. Hu B, Qiu-Lan H, Lei RE, Shi C, Jiang HX, Qin SY. Interleukin-9 promotes pancreatic cancer cells proliferation and migration via the miR-200a/ beta-catenin axis. Biomed Res Int. 2017;2017:2831056. 
10. Huang Q, Lei R, Qin Q, Qin S, Jiang H, Hu B. Interleukin-9 promotes pancreatic cancer cell proliferation and migration via activation of STAT3 pathway [in Chinese]. Xi Bao Yu Fen Zi Mian Yi Xue Za Zhi. 2017; 33:1228-1233.

11. Back TA, Chouin N, Lindegren S, et al. Cure of human ovarian carcinoma solid xenografts by fractionated [211At] alpha-radioimmunotherapy: Influence of tumor absorbed dose and effect on long-term survival. J Nucl Med. 2017;58(4):598-604.

12. Kashiwagi H, McDunn JE, Goedegebuure PS, et al. TAT-Bim induces extensive apoptosis in cancer cells. Ann Surg Oncol. 2007;14(5): 1763-1771.

13. Hawkins WG, Gold JS, Dyall R, et al. Immunization with DNA coding for gp100 results in CD4 T-cell independent antitumor immunity. Surgery. 2000;128(2):273-280.

14. Li HJ, Sun QM, Liu LZ, et al. High expression of IL-9R promotes the progression of human hepatocellular carcinoma and indicates a poor clinical outcome. Oncol Rep. 2015;34(2):795-802.

15. Banerjee $\mathrm{K}$, Resat $\mathrm{H}$. Constitutive activation of STAT3 in breast cancer cells: A review. Int J Cancer. 2016;138(11):2570-2578.

16. Makohon-Moore A, lacobuzio-Donahue CA. Pancreatic cancer biology and genetics from an evolutionary perspective. Nat Rev Cancer. 2016;16(9):553-565.

17. Laheru D, Jaffee EM. Immunotherapy for pancreatic cancer: Science driving clinical progress. Nat Rev Cancer. 2005;5(6):459-467.

18. Hoelzinger DB, Dominguez AL, Cohen PA, Gendler SJ. Inhibition of adaptive immunity by IL9 can be disrupted to achieve rapid T-cell sensitization and rejection of progressive tumor challenges. Cancer Res. 2014;74(23):6845-6855.

19. Parrot T, Allard M, Oger R, et al. IL-9 promotes the survival and function of human melanoma-infiltrating CD4(+) CD8(+) double-positive T cells. Eur J Immunol. 2016;46(7):1770-1782.

20. Ye ZJ, Zhou Q, Yin W, et al. Differentiation and immune regulation of IL-9-producing CD4 ${ }^{+}$T cells in malignant pleural effusion. Am J Respir Crit Care Med. 2012;186(11):1168-1179.
21. Smith SE, Hoelzinger DB, Dominguez AL, Van Snick J, Lustgarten J. Signals through 4-1BB inhibit T regulatory cells by blocking IL-9 production enhancing antitumor responses. Cancer Immunol Immunother. 2011;60(12):1775-1787.

22. Partecke LI, Sendler M, Kaeding A, et al. A syngeneic orthotopic murine model of pancreatic adenocarcinoma in the C57/BL6 mouse using the Panc02 and 6606PDA cell lines. Eur Surg Res. 2011;47(2): 98-107.

23. Mace TA, Shakya R, Pitarresi JR, et al. IL- 6 and PD-L1 antibody blockade combination therapy reduces tumour progression in murine models of pancreatic cancer. Gut. 2018;67(2):320-332.

24. Arpin CC, Mac S, Jiang Y, et al. Applying small molecule signal transducer and activator of transcription-3 (STAT3) protein inhibitors as pancreatic cancer therapeutics. Mol Cancer Ther. 2016;15(5):794-805.

25. Zimmers TA, Fishel ML, Bonetto A. STAT3 in the systemic inflammation of cancer cachexia. Semin Cell Dev Biol. 2016;54:28-41.

26. Yu H, Lee H, Herrmann A, Buettner R, Jove R. Revisiting STAT3 signaling in cancer: New and unexpected biological functions. Nat Rev Cancer. 2014;14(11):736-746.

27. Corcoran RB, Contino G, Deshpande V, et al. STAT3 plays a critical role in KRAS-induced pancreatic tumorigenesis. Cancer Res. 2011;71(14): 5020-5029.

28. Goswami R, Kaplan MH. A brief history of IL-9. J Immunol. 2011;186(6): 3283-3288.

29. Hornakova T, Staerk J, Royer Y, et al. Acute lymphoblastic leukemiaassociated JAK1 mutants activate the Janus kinase/STAT pathway via interleukin-9 receptor alpha homodimers. J Biol Chem. 2009;284(11): 6773-6781.

30. Liu X, Lv Z, Zou J, et al. Elevated AEG-1 expression in macrophages promotes hypopharyngeal cancer invasion through the STAT3MMP-9 signaling pathway. Oncotarget. 2016;7(47):77244-77256.

31. Middleton K, Jones J, Lwin Z, Coward JI. Interleukin-6: An angiogenic target in solid tumours. Crit Rev Oncol Hematol. 2014;89(1):129-139. 